\title{
GSNOR regulates cardiomyocyte differentiation and maturation through protein S-nitrosylation
}

\author{
Zachary W. Grimmett ${ }^{1,2}$, Nicholas M. Venetos ${ }^{2,3}$, Richard T. Premont ${ }^{2,4}$, Jonathan S. Stamler ${ }^{2,4}$ \\ 'Department of Pathology, Case Western Reserve University School of Medicine, Cleveland, OH 44106, USA. \\ ${ }^{2}$ Institute for Transformative Molecular Medicine, Department of Medicine, Case Western Reserve University School of \\ Medicine, Cleveland, OH 44106, USA. \\ ${ }^{3}$ Department of Biochemistry, Case Western Reserve University School of Medicine, Cleveland, OH 44106, USA. \\ ${ }^{4}$ Harrington Discovery Institute, University Hospitals Cleveland Medical Center, Cleveland, OH 44106, USA.
}

\begin{abstract}
Correspondence to: Dr. Jonathan S. Stamler, Institute for Transformative Molecular Medicine, Department of Medicine, Case Western Reserve University School of Medicine, 9501 Euclid Ave, Cleveland, OH 44106, USA; Harrington Discovery Institute, University Hospitals Cleveland Medical Center, 11100 Euclid Ave, Cleveland, OH 44106, USA. E-mail: jss156@case.edu
\end{abstract}

\begin{abstract}
How to cite this article: Grimmett ZW, Venetos NM, Premont RT, Stamler JS. GSNOR regulates cardiomyocyte differentiation and maturation through protein S-nitrosylation. J Cardiovasc Aging 2021;1:16. https://dx.doi.org/10.20517/jca.2021.25
\end{abstract}

Received: 29 Sep 2021 Accepted: 30 Sep 2021 Available online: 13 Oct 2021

Academic Editor: AJ Marian Copy Editor: Xi-Jun Chen Production Editor: Xi-Jun Chen

S-nitrosoglutathione reductase (GSNOR) is a denitrosylase enzyme responsible for reverting protein Snitrosylation (SNO). In this issue, Salerno et al. ${ }^{[1]}$ provide evidence that GSNOR deficiency - and thus elevated protein S-nitrosylation - accelerates cardiomyocyte differentiation and maturation of induced pluripotent stem cells (iPSCs). GSNOR inhibition (GSNOR ${ }^{-1}$ iPSCs) expedites the epithelial-mesenchymal transition (EMT) and promotes cardiomyocyte progenitor cell proliferation, differentiation, and migration. These findings are consistent with emerging roles for protein S-nitrosylation in developmental biology (including cardiomyocyte development), aging/longevity, and cancer.

\section{GSNOR-DEPENDENT S-NITROSYLATION REGULATES GSK-3 $\beta$ FUNCTIONS}

GSNOR modulates dynamic denitrosylation of hundreds of protein substrates (among > 20,000 in the published literature ${ }^{[2]}$ ) in response to a variety of biological stimuli ${ }^{[3]}$; Salerno et al. ${ }^{[1]}$ add GSK-3 $\beta$ (glycogen

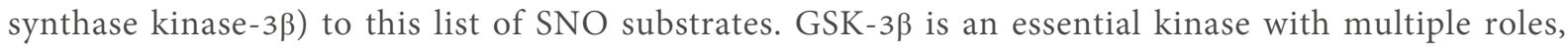
particularly in cardiovascular physiology ${ }^{[4]}$. Recently, GSK-3 $\beta$ has been shown to be S-nitrosylated at three major sites (Cys76, Cys199, and Cys317) and multiple minor sites, leading to inhibition of cytosolic kinase 
activity (with subsequent translocation to the nucleus and phosphorylation of nuclear targets - in lieu of cytoplasmic targets) $)^{[5]}$. S-nitrosylation of GSK-3 $\beta$ inhibits kinase activity independent of canonical inhibitory Ser9 phosphorylation, providing a novel locus of regulation for this important kinase. Salerno et al. ${ }^{[1]}$ innovatively demonstrate that S-nitrosylation of GSK-3 $\beta$ is enzymatically regulated by GSNOR in iPSCs during early cardiomyocyte differentiation. Further, they show that GSNOR ${ }^{-1}$ iPSCs - with elevated Snitrosylation - undergo accelerated cardiomyogenesis, including expedited differentiation, proliferation, migration, and EMT compared to wild-type iPSCs. SNO-GSK-3 $\beta$ may influence many of these processes, particularly EMT, which is promoted by three transcription factors (Snail, Slug, and Twist) already known to be regulated by GSK-3 $\beta^{[6]}$. The authors correlate elevated SNO-GSK-3 $\beta$ levels with accelerated cardiomyogenesis in GSNOR ${ }^{-1}$ iPSCs. Limitations of the study include that the authors did not identify the GSNOR-regulated SNO site(s) on GSK-3 $\beta$ in their model, nor did they perform studies demonstrating a causal role for SNO-GSK-3 $\beta$ in promoting cardiomyocyte differentiation (e.g., wild-type $v s$. SNO-site mutant GSK-3 $\beta$ restoration in knockout iPSCs). Indeed, no published study has yet investigated functional consequences of individual GSK-3ß Cys residue S-nitrosylation, which are likely tightly regulated by specific nitrosylase and denitrosylase enzymes in vivo ${ }^{[7]}$. Thus, in vitro treatment with exogenous NO donors $\left(\mathrm{GSNO}^{[1]}, \mathrm{CysNO}^{[5]}\right.$, etc.) may not always recapitulate physiology. Therefore, further confirmatory studies identifying the major site(s) of SNO regulation of GSK-3 $\beta$ relevant to cardiomyocyte maturation, including utilizing single Cys-mutant GSK-3 $\beta$ constructs combined with functional analyses, would be an illuminating area of future research.

GSNOR-dependent SNO-targets beyond GSK-3 $\beta$ may contribute to altered cardiomyocyte differentiation, and denitrosylases other than GSNOR may play important roles. GSNOR is one of seven known denitrosylases whose purview and targets are largely unexplored. Furthermore, many additional SNOregulatory enzymes remain to be discovered, including S-nitrosylases (SNO synthases and transnitrosylases) and denitrosylases, including but not limited to GSNOR subtypes; in other words, the multiplex enzyme systems ${ }^{[7]}$ regulating protein S-nitrosylation in cardiomyocytes have yet to be fully uncovered. This complexity elevates the impact of the findings presented by Salerno et al ${ }^{[1]}$, because it links cardiomyocyte differentiation specifically to GSNOR, one of multiple denitrosylase enzymes. In future studies it will be intriguing to explore what role other SNO-processing enzymes may plausibly play in cardiac regeneration and within the purview of stem cell maintenance and proliferation more broadly. Most notably, the SCoR/AKR1a1 denitrosylase has been implicated in metabolic reprogramming that may promote cancer and cellular proliferation ${ }^{[8]}$, and nitrosylase enzymes such as Hcp analogues ${ }^{[7]}$ may have important regulatory functions in these systems, which are entirely unexplored.

\section{S-NITROSYLATION IMPACTS MULTIPLE PATHWAYS TO REGULATE CARDIOVASCULAR PHYSIOLOGY}

Nitric oxide has long been deemed cardioprotective, particularly in the context of ischemia/reperfusion injury ${ }^{\left[{ }^{1}\right.}$. Many proteins (including RyR2, HIF-1 $\alpha$, SERCA2, hemoglobin, matrix metalloproteinase 9, complex 1 , etc ${ }^{[3]}$ ) have been shown to be S-nitrosylated in the heart under various conditions - regulating diverse signaling pathways including hypoxic responses, apoptosis, calcium handling/arrhythmogenesis, and microvascular control, among many others ${ }^{[1]}$. Functionally, protein S-nitrosylation exerts cardioprotective effects through diverse mechanisms, including induction of ischemic preconditioning and antioxidative defenses ${ }^{[9]}$. Though S-nitrosylation was originally thought to be broadly cardioprotective $e^{[9]}$, recent advances have exposed roles in cardiac pathology. For example, previous work by this laboratory has linked GSNOR to altered adrenergic responses in the heart and periphery ${ }^{[10]}$. GSNOR ${ }^{-/}$mice, in addition to exhibiting reduced peripheral vascular tone, demonstrate impaired $\beta$-agonist-induced inotropy; because a hallmark of heart failure (with reduced ejection fraction) is loss of inotropic reserve, elevated SNO-protein 
level is evidently pathologic in this context. Accumulating evidence also points to a causal role for SNOproteins in heart failure with preserved ejection fraction. By contrast, findings presented by Salerno et al. ${ }^{[1]}$ ascribe a beneficial role to elevated SNO-proteins in the heart, at least during early differentiation and maturation. This apparent contradiction reveals a more nuanced role for S-nitrosylation in cardiac physiology - one that is likely dependent on differentiation state, cell type, pathology, presence or absence of $\beta$-adrenergic stimulation, etc., and in which both over- and under-production of SNO-proteins is pathologic. In this issue, Salerno et al. ${ }^{[1]}$ describe a beneficial role for $\mathrm{GSNOR}^{-1}$ in cardiac development, plausibly dependent upon elevated SNO-GSK-3 $\beta$. Inhibition of GSK-3 $\beta$ promotes DNA synthesis, cell cycle re-entry and proliferation ${ }^{[1]}$, which may well explain the accelerated iPSC-derived cardiomyocyte development that they observe ${ }^{[1]}$. Unraveling this intricate relationship between S-nitrosylation and cardiovascular physiology will require attention to distinct SNO targets in different pathophysiological contexts.

\section{GSNOR AND S-NITROSYLATION: IMPACT ON DIFFERENTIATION AND IMPLICATIONS FOR AGING/LONGEVITY}

This study also sheds light on the influence of protein S-nitrosylation in stem cell biology. Maintenance of stem cell pluripotency, as well as the process of differentiation, are both regulated by NO; low NO levels suppress differentiation - marked by Nanog, Oct4, and Sox2 levels ${ }^{[12]}$ - while high concentrations of NO induce differentiation ${ }^{[13]}$. "Low" and "high" NO likely reflect different SNO targets. Indeed, Salerno et al. ${ }^{[1]}$ observe that $\mathrm{GSNOR}^{-1-}$ downregulates both Oct4 and Sox2, consistent with accelerated differentiation via "high NO". In corroborating work ${ }^{[14]}$, epigenetic downregulation of GSNOR appears to drive aging and cell senescence, with a reduction in GSNOR expression evident both in primary cells undergoing senescence as well as in aging mice and humans throughout their respective lifespans. Intriguingly, GSNOR ${ }^{-1}$ mice also demonstrate features overlapping those of experimental aging models, including impaired DNA repair, deficient osteogenesis, and neuromuscular dysfunction ${ }^{[14]}$. Indeed, GSNOR ${ }^{-/}$mice show nitrosative stress in conjunction with mitochondrial dysfunction, due at least in part to S-nitrosylation of specific target proteins (i.e., Drp1 and Parkin) that regulate mitochondrial dynamics and mitophagy ${ }^{[14]}$. Together, these data suggest that the role and function of GSNOR may vary with the age of the organism, in different organs, and across health and disease.

In a recent and exciting advance by Yi et al. ${ }^{[15]}$, GSNOR deficiency has also been shown to lead to abnormal hematopoietic stem cell (HSC) regeneration. In response to chemotherapy-induced injury, GSNOR ${ }^{-1} \mathrm{HSCs}$ demonstrate impaired self-renewal and thus diminished reconstitutive capacity relative to wild-type HSCs; this deficit is prevented by inhibition of NO synthesis. Thus, GSNOR-dependent regulation of SNO-protein levels appears to promote maintenance of not only iPSC pluripotency ${ }^{[1]}$ but also HSC self-renewal (and thus the ability to reconstitute the entire hematopoietic system). Unfortunately, Yi et al. ${ }^{[15]}$ do not identify SNOprotein substrates of GSNOR that regulate HSC self-renewal - a compelling research direction for future studies investigating aging and longevity.

Utilizing a dataset containing approximately 25,000 SNO sites identified in $>10,000$ proteins compiled by our laboratory, we find that many proteins previously identified as relevant to aging/longevity signaling pathways $^{[16]}$ are S-nitrosylated [Figure 1], though functional outcomes of this S-nitrosylation remain uncharacterized for most targets. Excitingly, Figure 1 contains only proteins identified in the plasma of test subjects; there are therefore many additional S-nitrosylated targets relevant to the aging process that are not among these 651 plasma proteins, including GSK-3 $\beta$. In cardiac cells specifically, hundreds of proteins are involved in aging/longevity and pluripotency/differentiation, and further characterization of SNO-GSK-3 $\beta$ may expose distinct consequences of this modification in the context of aging and longevity. For example, 


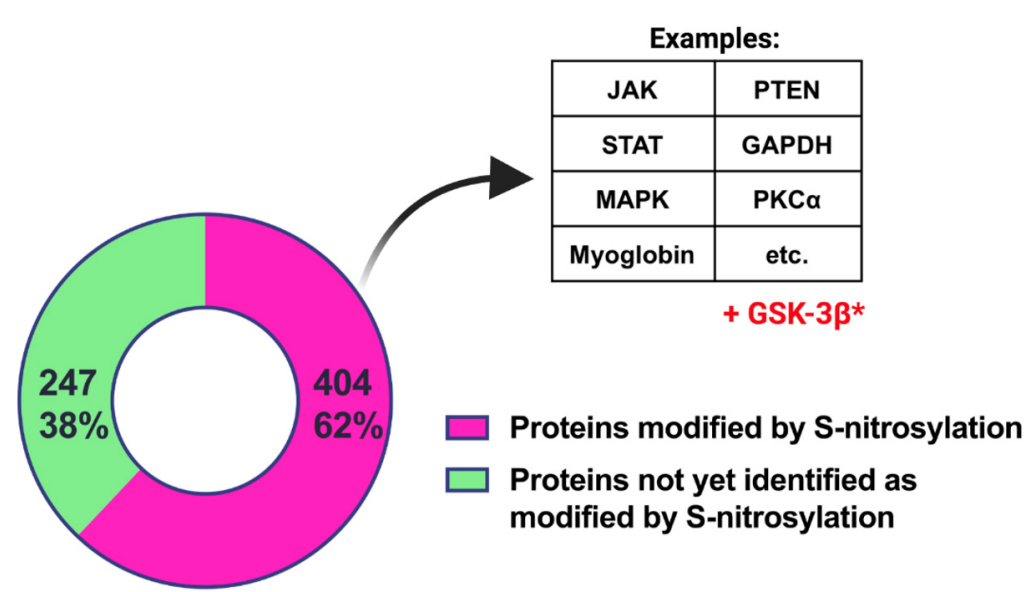

Total proteins: 651

Figure 1. S-nitrosylation modifies a majority of proteins related to aging and longevity. Of 651 plasma proteins identified as significantly associated with age (either over-or under-represented in aged individuals) ${ }^{[16]}, 404$ are known to be S-nitrosylated. Notable examples are displayed in the table to the right. A new addition, GSK-3ß, has been identified by Salerno et al. ${ }^{[1]}$ in this issue.

GSK-3 $\beta$ is known to negatively regulate the transcriptional coactivator PGC- $1 \alpha$, which influences many aspects of energy metabolism ${ }^{[17]}$. One such target of PGC- $1 \alpha$ is mitochondrial biogenesis - dysfunction of which has been implicated in aging. Indeed, upregulation of PGC-1 $\alpha$ in Drosophila is associated with enhanced mitochondrial biogenesis and increased lifespan ${ }^{[17]}$. Thus, SNO-mediated inhibition of GSK-3 $\beta$ might provide an avenue to upregulate PGC-1 $\alpha$ and enhance lifespan, which may be counterbalanced by excessive S-nitrosylation of mitochondrial proteins wherein GSNOR deficiency accelerates cellular aging and senescence ${ }^{[1,1,1]}$.

It thus appears likely that proper regulation of protein S-nitrosylation - as opposed to the absolute quantity of $\mathrm{NO} / \mathrm{SNO}$ - is deficient in aging, and thus, conversely, may drive longevity. Studies aiming to develop therapies focused on maintaining proper regulation of protein S-nitrosylation, particularly GSNORregulated S-nitrosylation, throughout the aging process may be illuminating and impactful in enhancing the longevity of organ systems. Additionally, unbiased mass-spectrometry-based studies identifying Snitrosylated cardiac proteins that regulate differentiation and maturation will be informative for future regenerative therapies, as no such dataset currently exists. Ideally, this valuable data will prove instructive for improving clinical modulation of cardiac regeneration post-injury, and will advance our scientific understanding of cardiac differentiation with the ultimate goal of improving cardiac tissue viability.

\section{DECLARATIONS}

\section{Authors' contributions}

Conceptualization: Grimmett ZW, Stamler JS

Data analysis: Grimmett ZW, Premont RT

Funding acquisition: Stamler JS

Resources: Stamler JS

Supervision: Premont RT, Stamler JS

Writing - original draft: Grimmett ZW

Writing - review and editing: Venetos NM, Premont RT, Stamler JS 


\section{Availability of data and materials}

Not applicable.

\section{Financial support and sponsorship}

Funding was provided by NIH grants HL075443, HL128192, HL126900, T32 GM007250, T32 HL134622-5 and DK119506, and American Heart Association-Allen Brain Health Initiative grant 19PABHI34580006. The content is solely the responsibility of the authors and does not necessarily represent the official views of the National Institutes of Health or other funders.

\section{Conflicts of interest}

Stamler JS has patents relating to S-nitrosylation and is a founder of SNOBio. CWRU and UHCMC have management plans in place. The other authors declared that there are no conflicts of interest.

\section{Ethical approval and consent to participate}

Not applicable.

\section{Consent for publication}

Not applicable.

\section{Copyright}

(c) The Author(s) 2021.

\section{REFERENCES}

1. Salerno AG, Wanschel ACBA, Dulce RA, Hatzistergos KE, Balkan W, Hare JM. S-nitrosoglutathione reductase (GSNOR) deficiency accelerates cardiomyocyte differentiation of induced pluripotent stem cells. J Cardiovasc Aging 2021;1:13. DOI

2. Li S, Yu K, Wu G, et al. pCysMod: prediction of multiple cysteine modifications based on deep learning framework. Front Cell Dev Biol 2021;9:617366. DOI PubMed PMC

3. Lima B, Forrester MT, Hess DT, Stamler JS. S-nitrosylation in cardiovascular signaling. Circ Res 2010;106:633-46. DOI PubMed PMC

4. Zhou HL, Stomberski CT, Stamler JS. Cross talk between S-nitrosylation and phosphorylation involving kinases and nitrosylases. Circ Res 2018;122:1485-7. DOI PubMed PMC

5. Wang SB, Venkatraman V, Crowgey EL, et al. Protein S-nitrosylation controls glycogen synthase kinase $3 \beta$ function independent of its phosphorylation state. Circ Res 2018;122:1517-31. DOI PubMed PMC

6. Zhou BP, Deng J, Xia W, et al. Dual regulation of Snail by GSK-3beta-mediated phosphorylation in control of epithelial-mesenchymal transition. Nat Cell Biol 2004;6:931-40. DOI PubMed

7. Seth D, Hess DT, Hausladen A, Wang L, Wang YJ, Stamler JS. A multiplex enzymatic machinery for cellular protein S-nitrosylation. Mol Cell 2018;69:451-64.e6. DOI PubMed PMC

8. Zhou HL, Zhang R, Anand P, et al. Metabolic reprogramming by the S-nitroso-CoA reductase system protects against kidney injury. Nature 2019;565:96-100. DOI PubMed PMC

9. Sun J, Murphy E. Protein S-nitrosylation and cardioprotection. Circ Res 2010;106:285-96. DOI PubMed PMC

10. Beigi F, Gonzalez DR, Minhas KM, et al. Dynamic denitrosylation via S-nitrosoglutathione reductase regulates cardiovascular function. Proc Natl Acad Sci U S A 2012;109:4314-9. DOI PubMed PMC

11. Uosaki H, Magadum A, Seo K, et al. Identification of chemicals inducing cardiomyocyte proliferation in developmental stage-specific manner with pluripotent stem cells. Circ Cardiovasc Genet 2013;6:624-33. DOI PubMed PMC

12. Tejedo JR, Tapia-Limonchi R, Mora-Castilla S, et al. Low concentrations of nitric oxide delay the differentiation of embryonic stem cells and promote their survival. Cell Death Dis 2010;1:e80. DOI PubMed PMC

13. Mora-Castilla S, Tejedo JR, Hmadcha A, et al. Nitric oxide repression of Nanog promotes mouse embryonic stem cell differentiation. Cell Death Differ 2010;17:1025-33. DOI PubMed

14. Rizza S, Cardaci S, Montagna C, et al. S-nitrosylation drives cell senescence and aging in mammals by controlling mitochondrial dynamics and mitophagy. Proc Natl Acad Sci U S A 2018;115:E3388-97. DOI PubMed PMC

15. Yi W, Zhang Y, Liu B, et al. Protein S-nitrosylation regulates proteostasis and viability of hematopoietic stem cell during regeneration. Cell Rep 2021;34:108922. DOI PubMed

16. Tanaka T, Basisty N, Fantoni G, et al. Plasma proteomic biomarker signature of age predicts health and life span. Elife 2020;9:e61073. DOI PubMed PMC

17. Gureev AP, Shaforostova EA, Popov VN. Regulation of mitochondrial biogenesis as a way for active longevity: interaction between the Nrf2 and PGC-1 $\alpha$ signaling pathways. Front Genet 2019;10:435. DOI PubMed PMC 